\title{
PRODUCTION-INVENTORY SYSTEM WITH FINITE PRODUCTION RATE, STOCK-DEPENDENT DEMAND, AND VARIABLE HOLDING COST
}

\author{
Hesham K. Alfares ${ }^{1}$
}

\begin{abstract}
In general, traditional production-inventory systems are based on a number of simplifying - but somewhat unrealistic - assumptions, including constant demand rate, constant holding cost, and instantaneous order replenishment. These assumptions have been individually challenged in numerous variations of production-inventory models. Finite production rate models, such as economic production quantity (EPQ) systems consider gradual order replenishment. Stockdependent demand models assume the demand rate to be an elastic function of the inventory level. Variable holding cost models assume the holding cost per unit per time period to be a function of the time spent in storage. In this paper, the three simplifying assumptions are simultaneously relaxed in a new production-inventory system with a finite production rate, stock-level dependent demand rate, and variable holding cost. Mathematical models and optimum solution procedures, including nonlinear programming, are presented for two functional forms of holding cost variability. The main contribution of this paper is the formulation and solution of a new production-inventory model that more closely represents real-world situations. The realistic assumptions and efficient solution algorithms should make the model practical and useful for industrial applications.
\end{abstract}

Keywords. Production-inventory system, stock-dependent demand, variable holding cost, nonlinear programming.

Mathematics Subject Classification. 90B05, 90B30, 90C30.

Received May 18, 2013. Accepted October 30, 2013.

1 P.O. Box 5067, Systems Engineering Department, King Fahd University of Petroleum and Minerals, Dhahran 31261, Saudi Arabia. alfares@kfupm.edu.sa 


\section{INTRODUCTION}

Traditional production-inventory control systems, such as the economic order quantity (EOQ) model, are usually based on the assumptions of constant demand rate, constant inventory carrying cost, and instantaneous order arrival. This paper presents a realistic production-inventory model in which all these assumptions are relaxed. The EOQ assumption of instantaneous order arrival means the whole order quantity (i.e., all units of the purchased lot) is received at once from the supplier. This assumption is not valid if these units are manufactured and received over an extended time interval. The economic production quantity (EPQ) model relaxes this assumption by incorporating a gradual order receipt, i.e. a finite production rate. This paper presents a production-inventory control system with a finite production rate, a stock-dependent demand rate, and a variable holding cost.

Demand variability according to item availability and price is a frequently observed phenomenon. In stock-dependent demand models, the demand for a given item increases with higher item availability. A bigger item display tends to attract the attention of more customers, leading to increased sales. Customers may view larger item stock as an indication of the item's popularity and also as a sign of reliable, continuing supply. Furthermore, a larger inventory leads to more on-time delivery and fewer shortages, therefore improving customer satisfaction and stimulating additional demand. Stock-dependent demand is a widely accepted concept in the literature, as illustrated in the Urban [22] survey. Several studies provide strong evidence to support this concept. For example, Koschat [11] empirically confirms the existence of a positive correlation between demand for a given product and its on-shelf inventory. Since higher sales are made on the expense of carrying more stock, firms need to find the optimum balance between the extra holding cost and the additional profit resulting from the induced sales.

In inventory models with variable holding costs, the holding cost is a function of either the storage level or the storage time. Holding cost time variability is used to reflect the fact that longer storage times frequently require higher holding costs. Longer storage periods, especially for perishable products, usually require more expensive specialized storage facilities. Time-dependent holding cost models represent holding cost either as a continuous nonlinear function or as a discontinuous step function of storage time. In continuous nonlinear functions, the unit holding cost gradually increases with longer storage time. However, in discontinuous step functions, the storage time is divided into few intervals, with one unit holding cost rate for each interval. Therefore, the unit holding cost is constant within each interval, but it immediately jumps to the next rate (step) as soon as the storage time extends to the next interval.

The aim of this paper is to present a production-inventory model with a finite production rate, a stock-level dependent demand rate, and a variable holding cost. In this model, the demand rate is an increasing power function of the current inventory level, and the holding cost is an increasing step function of the time spent 
in storage. Two types of holding cost step functions are considered: retroactive holding cost, and incremental holding cost.

Subsequent sections of this paper are organized as follows. Relevant literature is reviewed in Section 2. The inventory model is presented and analyzed in Section 3. The model is formulated and the optimal solution process is developed for retroactive and incremental holding costs in Sections 4 and 5, respectively. Finally, results are discussed and conclusions are provided in Section 6.

\section{Literature REVIEW}

Urban [22] provides a comprehensive review of inventory models with stockdependent demand rates published up to 2004, classifying work in this area into two main types. In the first type, pioneered by Gupta and Vrat [10], the demand rate is a function of the initial inventory. In the second type, pioneered by Baker and Urban $[2,3]$, the demand rate is a function of the current inventory. A third type could be considered as models in which the demand has two stages, an initial period of level-dependent demand followed by a period of constant demand. In this section, we review several categories of recent production-inventory models with variable holding costs and stock-dependent demand rates.

\subsection{EOQ-TYPE MODELS WITH STOCK-DEPENDENT DEMAND}

In inventory models with variable demand rates, the demand is either a function of time or a function of the stock level. According to Urban [22], demand dependence on the stock level has several functional forms, including: linear, power, and posynomial. Several models have been proposed for deteriorating items with stock-level dependent demand rates. Min and Zhou [12] develop an EOQ model for perishable items with a stock-dependent demand rate. Unsatisfied demands are partially backlogged, and the demand rate during shortages depends on the negative inventory level, i.e. the amount of shortage. Sana et al. [16] present EOQ and EPQ inventory models in which the demand rate depends on three factors: stock level, selling price, and advertising. Both deteriorating and ameliorating items are considered, and the objective is to maximize average profit subject to budget and storage capacity constraints. Yang et al. [25] propose an EOQ model for deteriorating items with a stock-dependent demand rate, partial backlogging, inflation, varying replenishment cycles, and varying shortage intervals. Unique optimal solutions are shown to exist, and good heuristic solution procedures are developed.

\subsection{EOQ-TYPe MODELS WITH VARIABLE HOLDING COST}

An increasing number of EOQ-type inventory models assume variable and nonlinear holding costs. Muhlemann and Valtis-Spanopoulos [14] are the first to introduce variable holding costs into the EOQ model. In their EOQ-type model, the holding cost is assumed to be a function of the average inventory value. Their justification is that the cost of financing increases as the amount of investment (value 
of the inventory) increases. Weiss [24] develops deterministic and stochastic EOQ models in which the per-unit holding cost is a non-linear function of the storage duration. According to Weiss [24], this assumption is applicable to inventory systems where the value of stored items decreases non-linearly with storage length. For the deterministic demand case, formulas are developed for the optimal order quantity under both finite and infinite horizons. For the stochastic demand case, a procedure to determine the optimal order quantity is described and computational results are presented. Ferguson et al. [4] reexamine the Weiss [24] model in which the cumulative holding cost is a nonlinear function of time. This model is shown to be an approximation to the optimal order quantity for perishable grocery items subject to delivery fees, where discounts are used to increase demand prior to the expiration dates. Given historical data, regression is used to estimate the parameters of the holding function from storage and capital costs, product lifetime, and discount policy.

\subsection{EOQ-TYPe MODEls With VARIABLE DEMAND AND VARIABLE HOLDing COST}

Goh [9] presents the first inventory model in which the demand is stock dependent and the holding cost varies with storage duration. Goh considers two types of holding cost variation: (a) a nonlinear function of storage time, and (b) a nonlinear function of storage level. For both cases, optimal ordering policies are developed for the deterministic, single-item, and infinite-horizon inventory model. Giri et al. [7] present a generalized EOQ-type model for deteriorating items, in which the demand rate, the deterioration rate, the ordering cost, and the holding cost are continuous functions of time. Assuming completely backlogged shortages, the optimal ordering policy is developed for a finite planning horizon. Giri and Chaudhuri [6] develop another EOQ-type model for deteriorating items in which the demand rate is a function of the stock level. The holding cost is assumed to be a continuous nonlinear function of either the stock level or the storage duration, and approximate optimal solutions are derived for the two cases. The assumption of increasing holding cost with longer storage is applicable to deteriorating items that require increasingly better storage facilities to avoid deterioration.

Teng and Yang [20] generalize the EOQ model to consider a fluctuating demand rate, time-varying unit cost, and generalized holding cost. The holding cost is assumed to include both size-related parts (such as the warehouse cost) and valuerelated parts (such as the insurance cost). The cost function is shown to be convex, and a simple heuristic solution algorithm is proposed. Roy [15] formulates two EOQ models for deteriorating items in which the demand rate is a function of the selling price. Both the deterioration rate and the holding cost are continuous, linearly increasing functions of storage duration. Shortages are allowed and are completely backlogged in the first model, while they are not allowed in the second model. 
Gayen and Pal [5] analyze a two-warehouse inventory model for a deteriorating item, in which both the demand rate and the holding cost are assumed to be continuous power functions of the current inventory level. Procedures are provided for approximating the optimal order policy, and results of computational experiments are presented. Mahata and Goswami [13] investigate an EOQ model for deteriorating items, assuming a stock-dependent demand rate and a variable holding cost. The holding cost is considered as a non-linear function of either the length of storage time or the current inventory level. Since real-world problems are characterized by imprecise and/or incomplete cost information, the demand rate, holding cost, ordering cost, and unit purchase cost are considered to be fuzzy numbers. The objective function is shown to be convex, and algorithms are developed for determining the optimal ordering policies.

Alfares [1] presents a stock-dependent EOQ-type model with two types of holding cost discontinuous step functions. As the storage time extends to the next time period, the new (higher) holding cost can be applied either retroactively to all storage periods, or incrementally to the new period only. Urban [23] extends Alfares [1] work by allowing non-zero end inventory for each cycle, and shifting to a maximum-profit objective. Singh, Kumar, and Gaur [18] present an EOQ model with time-dependent demand and partial backlogging of unsatisfied demand. They also consider deteriorating items, inflation, and an incremental holding cost function. Approximate optimal solutions are provided to minimize the inflation-adjusted total cost of ordering, holding, purchasing, and backlogging.

\subsection{EPQ-TYPE MODELS WITH VARIABLE DEMAND AND/OR HOLDING COST}

Assuming constant holding cost, Goh [8] presents three models of stockdependent demand inventory systems: (a) an EOQ model with no shortages, (b) an EOQ model with shortages, and (c) an EPQ model. Sarfaraz [17] develops a modified EPQ model in which the holding cost is composed of two components: an investment cost proportional to the dollar value of inventory, and a capacity cost proportional to the maximum inventory level. Two cases are formulated and optimally solved: a deterministic demand model, and stochastic demand model that considers the costs of shortages and safety stocks. As stated earlier, Sana et al. [16] analyze an EPQ model with a stock-dependent demand rate and storage capacity limitations.

Tripathy et al. [21] formulate an EPQ model for deteriorating items, assuming the demand and the production rates are constant and the holding cost is a nonlinear continuous function of storage time. Partially deteriorated items are sold with a discount, while completely deteriorated items are discarded, and shortages are not allowed. Singh, Singh, and Vaish [19] develop an EPQ inventory model where demand is a linear function of time, and the production rate is a linear function of the demand. Two cases of holding costs are considered: (i) holding cost is constant, and (ii) holding cost is a continuous linear function of time. Optimal 
solutions are developed to minimize the total cost, including interest charges for payment delays.

The objective of this paper is to develop mathematical models and optimum solution procedures for two types of production-inventory systems with a stock-dependent demand rate, a finite production (replenishment) rate, and timedependent holding cost functions.

\section{Model Development}

\subsection{Notation}

The notation below is mostly adopted from Goh [8] and Alfares [1] for the production-inventory model to be developed:

$q(t)=$ quantity on-hand (inventory level) at time $t$,

$D=$ constant (base) demand rate,

$P=$ production rate during the first phase of the cycle $\left(0 \leq t \leq t_{1}\right)$,

$n=$ number of distinct time periods with different holding cost rates,

$t=$ time from the start of the cycle at $t=0$,

$t_{1}=$ end time of the first (uptime) phase of the cycle,

$\sigma_{i}=$ end time of holding-cost interval $i$, where $i=1,2, \ldots n, \sigma_{0}=0$, and $\sigma_{n}=\infty$,

$K=$ ordering/setup cost per order,

$h(t)=$ holding cost of the item at time $t, h(t)=h_{i}$ if $\sigma_{i-1} \leq t \leq \sigma_{i}$,

$T=$ cycle time, i.e. time between producing two consecutive orders of size $S$,

$\beta=$ demand elasticity rate in relation to the inventory level,

$Q=$ maximum inventory level, corresponding to time $t=t_{1}$,

$S=$ production lot size $=P t_{1}$.

\subsection{Assumption and limitations}

1. A single item with an infinite planning horizon is considered.

2. The holding cost is an increasing step function of storage duration $\left(h_{1}<h_{2}<\right.$ $\left.\ldots<h_{n}\right)$.

3. An order of lot size $S$ is produced gradually in $t_{1}$ periods at a constant rate $P$.

4. The units do not lose value during storage (no item deterioration).

5. Shortages are not allowed.

6. The demand rate $R$ is an increasing power function of the inventory level $q$, given as:

$$
R(q)=D q^{\beta}, \quad D>0, \quad 0<\beta<1, \quad q \geq 0 .
$$

\subsection{THE PRODUCTION-INVENTORY MODEL}

As shown in Figure 1, the inventory level variation over time $q(t)$ during a typical cycle is divided into two phases. During the first (uptime) phase of the cycle, a new order is produced at a constant rate $P$ while the inventory is consumed at the 


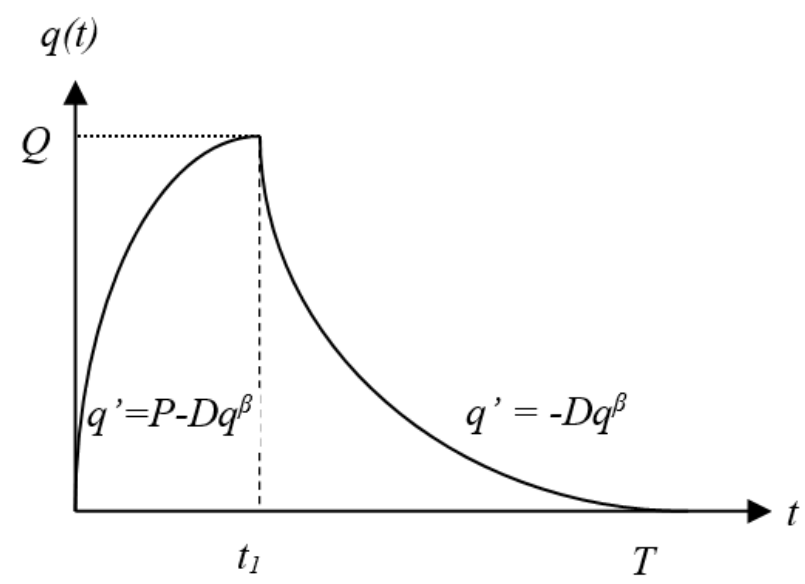

FiguRE 1. The inventory level during the two phases of the cycle.

stock-dependent demand rate of $D q^{\beta}$. Hence, the rate of change in the inventory level is expressed as follows:

$$
\frac{\mathrm{d} q(t)}{\mathrm{d} t}=P-D q(t)^{\beta}, \quad 0 \leq t \leq t_{1}, \quad P>D, \quad q(0)=0
$$

During the second (downtime) phase of the cycle, the rate of change (decrease) in the inventory level is equal to the demand rate, which is given by:

$$
\frac{\mathrm{d} q(t)}{\mathrm{d} t}=-D[q(t)]^{\beta}, \quad t_{1} \leq t \leq T, \quad q(T)=0
$$

The total cost of the system includes two components: the ordering cost, and the holding cost. Since one order at a cost $K$ is made in each cycle, the ordering cost per cycle is simply $K$. The holding cost per cycle is obtained by integrating the product of the holding cost $h(t)$ and the inventory level $q(t)$ for the whole cycle. However, the true objective is to minimize the total cost per unit time $T C$, which is obtained by dividing the total cost per cycle by the cycle time $T$. Therefore, the total cost per unit time $T C$ is given by:

$$
T C=\frac{K}{T}+\frac{1}{T} \int_{0}^{t_{1}} h(t) q(t) \mathrm{d} t+\frac{1}{T} \int_{t_{1}}^{T} h(t) q(t) \mathrm{d} t .
$$

During the uptime phase of the cycle $\left(0 \leq t \leq t_{1}\right)$, rearranging and integrating the ordinary differential equation (3.2) results in the following:

$$
\int_{0}^{t} \frac{1}{1-\alpha q(t)^{\beta}} \mathrm{d} q=P \int_{0}^{t} \mathrm{~d} t
$$

where

$$
\alpha=D / P
$$


The left-hand side of (3.5) can be integrated to yield the hyper-geometric function ${ }_{2} F_{1}$.

$$
\int \frac{1}{1-\alpha q^{\beta}} \mathrm{d} q=q \times{ }_{2} F_{1}\left(1, \frac{1}{\beta} ; 1+\frac{1}{\beta} ; \alpha q^{\beta}\right) .
$$

After simplification, we obtain:

$$
\left.\sum_{n=0}^{\infty} \frac{\alpha^{n} q(t)^{n \beta+1}}{(n \beta+1)}\right|_{0} ^{t}=P t
$$

Since $q(0)=0$ while $q\left(t_{1}\right)=Q$, integrating over the range $\left[0, t_{1}\right]$ gives:

$$
t_{1}=\sum_{n=0}^{\infty} \frac{\alpha^{n} Q^{n \beta+1}}{P(n \beta+1)} .
$$

In order to obtain an expression for $q(t)$ during the uptime phase, (3.2) is rearranged differently from (3.5) and integrated as follows:

$$
\int_{a}^{b} q(t) \mathrm{d} t=\frac{1}{P} \int_{a}^{b} \frac{q(t)}{1-\alpha q(t)^{\beta}} \mathrm{d} q .
$$

The right-hand side of (3.8) again integrates to the hyper-geometric function ${ }_{2} F_{1}$.

After simplification, (3.8) can be written as:

$$
\int_{a}^{b} q(t) \mathrm{d} t=\sum_{n=0}^{\infty} \frac{\alpha^{n}\left[q(b)^{n \beta+2}-q(a)^{n \beta+2}\right]}{P(n \beta+2)} .
$$

During the downtime phase of the cycle $\left(t_{1} \leq t \leq T\right)$, Alfares [1] provides the following solution of the ordinary differential equation (3.3):

$$
\begin{gathered}
\int_{a}^{b} q(t) \mathrm{d} t=\frac{q(a)^{2-\beta}-q(t)^{2-\beta}}{D(2-\beta)} \\
T=t_{1}+\frac{Q^{1-\beta}}{D(1-\beta)} .
\end{gathered}
$$

Substituting the appropriate terms from (3.7) and (3.9)-(3.11) into (3.4), and setting the derivative with respect to $Q$ equal to 0 , we solve for the optimum value of $Q$. Following the derivation steps described in Appendix A, the minimum total cost $T C$ is obtained by solving the following equation for $Q$ :

$$
h Q^{2-\beta}+h(1-\beta)(2-\beta)\left(\alpha Q^{2}\right) \sum_{n=0}^{\infty} \frac{\left(\alpha Q^{\beta}\right)^{n}}{(n \beta+1)(n \beta+2)}=K D(1-\beta)(2-\beta)
$$

where

$$
\alpha Q^{\beta}<1 \text {. }
$$


For convergence of the power series in (3.12), the feasibility condition $\left(\alpha Q^{\beta}<1\right)$ must be satisfied. To solve (3.12), the infinite power series is well approximated with a reasonably large number of terms. Based on extensive numerical experiments, it was found that including 2000 terms is quite sufficient for accurate results, even in the worst case when the value of $\alpha Q^{\beta}$ is nearly equal to 1.

From the optimum value of $Q$, the following quantities can be calculated:

$$
\begin{aligned}
\int_{0}^{T} q(t) \mathrm{d} t & =\frac{Q^{2-\beta}}{D(2-\beta)}+\sum_{n=0}^{\infty} \frac{\alpha^{n} Q^{n \beta+2}}{P(n \beta+2)} \\
S & =\sum_{0}^{\infty} \frac{\alpha^{n} Q^{n \beta+1}}{n \beta+1} .
\end{aligned}
$$

\section{Retroactive holding Cost}

Assuming a retroactive holding cost, only one holding cost is used. That is, the holding cost of the last storage interval is used retroactively for earlier intervals. Assuming that the cycle ends in interval e, $\left(\sigma_{e-1} \leq T \leq \sigma_{e}\right)$, then the rate $h_{e}$ is applied to holding cost intervals $1,2, \ldots, e$. Therefore, the total cost per unit time $T C$ is given by:

$$
T C=\frac{K}{T}+\frac{h_{i}}{T} \int_{0}^{T} q(t) \mathrm{d} t \quad \sigma_{i-1} \leq T \leq \sigma_{i}
$$

\subsection{SOlution PROCEDURE}

The optimum solution can be determined by using the following steps:

1. Starting with the lowest holding cost $h_{1}$, use (3.12) to determine $Q$ and (3.11) to determine $T$ for each $h_{i}$ until $Q$ is realizable (i.e., $\sigma_{i 1} \leq T \leq \sigma_{i}$ ). Call these values $T_{R}$ and $Q_{R}$.

2. Calculate all break-point values of $Q, Q_{i}=Q\left(\sigma_{i}\right)$, by setting $T=\sigma_{i}$ in (3.11) and solving for $Q_{i}$.

3. For all $Q_{R}$ and $Q_{i}$, use (3.13) to calculate and then substitute the result using the appropriate $h_{i}$ into (4.1) to calculate the total cost $T C$.

4. Choose the value of $Q$ that gives the lowest $T C$, and then use (3.14) to calculate the corresponding production lot size $S$.

\subsection{EXAMPLE 1}

Given the following parameters:

$D=400$ units per year,

$P=1000$ units per year,

$K=\$ 300$ per order,

$\beta=0.1$, 
$h_{1}=\$ 6 /$ unit/year, $h_{2}=\$ 8 /$ unit/year, $h_{3}=\$ 10 /$ unit $/$ year,

$\sigma_{1}=0.3$ years, $\sigma_{2}=0.6$ years, $\sigma_{3}=\infty$.

The solution procedure is implemented in the following steps.

Step 1. Substituting $h_{1}=6$ (for $0<T \leq 0.3$ ), solving (3.12) gives: $Q=155$.

Substituting $Q=155$ into (3.11) gives $T=0.656$ (not realizable).

Using $h_{2}=8$ (for $0.3 \leq T \leq 0.6$ ), solving (3.12) gives: $Q=135$.

Substituting $Q=135$ into (3.11) gives $T=0.567$ (realizable).

Step 2. Setting $T=\sigma_{1}=0.3$, solving (3.11) gives: $Q_{1}=73$.

Setting $T=\sigma_{2}=0.6$, solving (3.11) gives: $Q_{2}=142$.

Step 3. Substituting $Q_{R}=135$ and $h_{2}=8$ into (3.13) and (4.1) gives: $T C=$ $\$ 1,078.09$.

Substituting $Q_{1}=73$ and $h_{1}=6$ into (3.13) and (4.1) gives: $T C=$ $\$ 1,223.08$.

Substituting $Q_{2}=142$ and $h_{2}=8$ into (3.13) and (4.1) gives: $T C=$ $\$ 1,079.64$.

Step 4. The minimum cost $(T C=\$ 1,078.09 /$ year $)$ is obtained with $Q=135$. Using (3.14), the corresponding lot size is calculated as: $S=338$. Therefore, the optimum inventory policy is:

Cycle time $T=0.567$ year.

Lot size $S=338$.

\section{INCREMENTAL HOLDING COST}

An incrementally increasing holding cost means that a different holding cost $h_{i}$ applies to each storage interval $i\left(\sigma_{i 1} \leq t \leq \sigma_{i}\right)$. The objective, which is to minimize the total cost per unit time, is expressed as follows:

$$
\text { Minimize } T I C=\frac{K}{T}+\frac{1}{T} \sum_{i=1}^{e} h_{i} \int_{\tau_{i-1}}^{\tau_{i}} q(t) d t
$$

A given interval $\left[\sigma_{i-1}, \sigma_{i}\right]$ may fall entirely in the first phase (before $t_{1}$ ), entirely in the second phase (after $t_{1}$ ), or straddle both phases. Therefore, depending on the relationship between end points $\left[\sigma_{i-1}, \sigma_{i}\right]$ and $t_{1}$, there are three possible procedures for calculating the integrals in (5.1). If the interval falls entirely in the first phase $\left(\sigma_{i-1}<\sigma_{i} \leq t_{1}\right)$, then we use (3.9), replacing $a$ and $b$ by $\sigma_{i-1}$ and $\sigma_{i}$. If the interval falls entirely in the second phase $\left(t_{1} \leq \sigma_{i-1}<\sigma_{i}\right)$, then we use (3.10), replacing $a$ and $b$ by $\sigma_{i-1}$ and $\sigma_{i}$. However, if the interval straddles both phases $\left(\sigma_{i-1} \leq t_{1}<\sigma_{i}\right)$, then we have to evaluate the integral over $\left[\sigma_{i-1}, \sigma_{i}\right]$ using a combination of (3.9) and (3.10) as follows:

$\int_{\sigma_{i-1}}^{\sigma_{i}} q(t) \mathrm{d} t=\sum_{n=0}^{\infty} \frac{\alpha^{n}\left[Q^{n \beta+2}-q\left(\sigma_{i-1}\right)^{n \beta+2}\right]}{P(n \beta+2)}+\frac{Q^{2-\beta}-q\left(\sigma_{i}\right)^{2-\beta}}{D(2-\beta)}, \quad \sigma_{i-1} \leq t_{1}<\sigma_{i}$. 
The inventory level $q(t)$ is determined from (3.2) during the uptime phase and from (3.3) during the downtime phase. Therefore, the functions $q\left(\sigma_{i}\right)$ and $q\left(\sigma_{i-1}\right)$ are determined from two different expressions in the two phases.

$$
\begin{gathered}
\sum_{n=0}^{\infty} \frac{\alpha^{n} q(t)^{n \beta+1}}{(n \beta+1)}=P t, \quad 0 \leq \sigma_{i} \leq t_{1} \\
q(t)=\left[Q^{1-\beta}-D(1-\beta)\left(t-t_{1}\right)\right]^{\frac{1}{1-\beta}}, \quad t_{1} \leq \sigma_{i} \leq T
\end{gathered}
$$

If the current interval $i$ is the last interval in the cycle $(i . e . i=e$ ), then the intervals end point $\sigma_{i}=\sigma_{e}$ has to be replaced by the cycle time $T$. Therefore, it should be assumed that $\sigma_{i}=T$ and $q\left(\sigma_{i}\right)=0$ for the last interval $i$ in (5.1). Furthermore, the cycle time $T$ must fall in the last interval $e$.

$$
\sigma_{e-1} \leq T \leq \sigma_{e}
$$

\subsection{SOLUtion PROCEDURE}

Since the objective function (5.1) is constrained, direct optimization by differential calculus is not feasible. For the incremental holding cost case, the optimum solution is obtained by nonlinear programming according to the following steps:

1. Substitute the minimum and maximum values of $h_{i}$ in (3.12) to find the range of values for $\mathrm{Q}$, and then use (3.7) and (3.11) to determine the corresponding range of intervals for $t_{1}$ and $T$.

2. For each possible combination of $t_{1}$ and $T$ intervals, formulate a nonlinear programming (NLP) model whose objective function is (5.1), decision variable is $Q$, and constraints are (3.7), (3.11), and (5.3)-(5.5). In each NLP model, use the applicable terms for each interval $\left[\sigma_{i-1}, \sigma_{i}\right]$ in the objective and constraints.

3. For each combination of $t_{1}$ and $T$ intervals, solve the corresponding NLP model to find the optimum solution.

4. Choose the feasible solution with the minimum total cost $T C$.

\subsection{EXAMPLE 2}

Resolve Example 1 assuming the holding cost increases incrementally.

The solution procedure is implemented in the following steps.

Step 1. For $h_{1}=6(0<T \leq 0.3)$, (3.12), (3.7), and (3.11) give $Q=155$, $t_{1}=0.396$, and $T=0.656$.

For $h_{3}=10(T>0.6),(3.12),(3.7)$, and (3.11) give $Q=121, t_{1}=0.298$, and $T=0.506$.

Clearly $T$ falls either in the second or the third interval. However, although the results indicate that $t_{1}$ may fall either in the first or the second interval, the minimum $t_{1}=0.298$ is nearly in the second interval. Therefore, we will consider $t_{1}$ to belong to the second interval only. 
Step 2 (a). Assuming both $t_{1}$ and $T$ are in the second interval, then $e=2$. In the objective function, (3.9) is used for the first interval $(0<t \leq 0.3)$ which is in the uptime phase, while (5.2) is used for the second interval $(0.3<t \leq T)$ which straddles the uptime and downtime phases. Therefore, the NLP model is formulated as follows:

$$
\begin{aligned}
\text { Minimize } T I C= & \frac{300}{T}+\frac{6}{T} \sum_{n=0}^{\infty} \frac{0.4^{n} q(0.3)^{0.1 n+2}}{1000(0.1 n+2)} \\
& +\frac{8}{T}\left(\sum_{n=0}^{\infty} \frac{0.4^{n}\left[Q^{0.1 n+2}-q(0.3)^{0.1 n+2}\right]}{1000(0.1 n+2)}\right. \\
& \left.+\frac{Q^{2-0.1}}{400(2-0.1)}\right) .
\end{aligned}
$$

Subject to:

$$
\begin{aligned}
& \sum_{n=0}^{\infty} \frac{0.4^{n} q(0.3)^{0.1 n+1}}{(0.1 n+1)}=1000(0.3) \quad 0<\sigma_{i}<t_{1} \\
& t_{1}=\sum_{n=0}^{\infty} \frac{0.4^{n} Q^{0.1 n+1}}{1000(0.1 n+1)} \\
& T=t_{1}+\frac{Q^{1-0.1}}{400(1-0.1)} \\
& 0.3 \leq T \leq 0.6 .
\end{aligned}
$$

Step 2 (b). Assuming $t_{1}$ is in the second interval and $T$ is in the third interval, then $e=3$. The NLP model is formulated as follows:

$$
\begin{aligned}
\text { Minimize } T I C= & \frac{300}{T}+\frac{6}{T} \sum_{n=0}^{\infty} \frac{0.4^{n} q(0.3)^{0.1 n+2}}{1000(0.1 n+2)} \\
& +\frac{8}{T}\left(\sum_{n=0}^{\infty} \frac{0.4^{n}\left[Q^{0.1 n+2}-q(0.3)^{0.1 n+2}\right]}{1000(0.1 n+2)}\right. \\
& \left.+\frac{Q^{2-0.1}-q(0.6)^{2-0.1}}{400(2-0.1)}\right) \\
& +\frac{10}{T}\left(\frac{q(0.6)^{2-0.1}}{400(2-0.1)}\right) .
\end{aligned}
$$

This function is minimized subject to constraints (5.7)-(5.9) above, in addition to the two following constraints:

$$
\begin{aligned}
& q(0.6)=\left[Q^{1-0.1}-400(1-0.1)\left(0.6-t_{1}\right)\right]^{\frac{1}{1-0.1}} \\
& T>0.6 .
\end{aligned}
$$


Step 3. The optimum solution of the NLP in step 2(a) is:

$$
Q=126, t_{1}=0.312, T=0.528, S=312, T C=\$ 1,007.01 .
$$

The optimum solution of the NLP in step 2(b) is:

$$
Q=143, t_{1}=0.361, T=0.603, S=361, T C=\$ 1,015.62 .
$$

Step 4. The optimum solution of Example 2 for incremental holding cost is:

$$
Q=126, t_{1}=0.312, T=0.528, S=312, T C=\$ 1,007.01 .
$$

\section{Discussion AND CONClusions}

The two algorithms presented in sections 4 and 5 are quite efficient. In the retroactive case, the number of algorithm iterations is proportional to the number of holding cost rates, i.e., number of holding cost intervals, $n$. Therefore, the complexity of the retroactive holding cost algorithm is simply $O(n)$. In the incremental case, the number of algorithm iterations is proportional to the number of feasible combinations of $t_{1}$ and $T$ intervals. Let $u$ and $e$ respectively denote holding costs intervals corresponding to $t_{1}$ and $T$. Since $t_{1}$ cannot exceed $T$, then $u$ cannot exceed $e(u=1, \ldots, e)$, and since $e$ cannot exceed $n$, then the number of feasible combinations of $u$ and $e$ cannot exceed $\sum_{i=1}^{n} i=n(n+1) / 2$. Therefore, the complexity of the incremental holding cost algorithm is $O\left(n^{2}\right)$.

To effectively use the models and algorithms in real-life applications, concerned mangers need to validate that the models assumptions are applicable in their organizations. First, production managers have to confirm that the demand for the given product is indeed an increasing power function of the stock level. Subsequently, the parameters of this function $(D$ and $\beta$ ) need to be estimated by least-squares regression from historical inventory and demand data. In addition, the parameters of the holding cost function $\left(\sigma_{i}\right.$ and $\left.h_{i}\right)$ and its particular form (retroactive or incremental) have to be determined. For the solution of the equations and nonlinear programs, managers may conveniently use Microsoft Excel with Solver add-in.

This paper presented a production-inventory system with a finite production rate in which both the demand rate and the holding cost are variables. The demand rate is stock dependent, varying as an increasing power function of the instantaneous inventory level. The holding cost is time dependent, varying either retroactively or incrementally as an increasing step function of the storage duration. Optimization models and solution procedures have been developed, and examples have been solved. Several steps of nonlinear programming are used to solve problems with incremental holding cost srtucture. For future research, the work presented here can be extended in several directions, including variable production rates, variable ordering costs, and price-dependent demands. 


\section{Appendix A. Derivation of Equation (3.12)}

Equation (3.4) can be written as follows:

$$
T C=\frac{K}{T}+\frac{h}{T} \int_{0}^{t_{1}} q(t) \mathrm{d} t+\frac{h}{T} \int_{t_{1}}^{T} q(t) \mathrm{d} t .
$$

From (3.9), the uptime phase integral is:

$$
\int_{0}^{t_{1}} q(t) d t=\frac{Q^{2}}{P} \sum_{n=0}^{\infty} \frac{\alpha^{n} Q^{n \beta}}{(2+n \beta)} .
$$

From (3.10), the downtime phase integral is:

$$
\int_{t_{1}}^{T} q(t) \mathrm{d} t=\frac{Q^{2-\beta}}{D(2-\beta)} .
$$

Substituting (A.2) and (A.3) and the values of $t_{1}$ and $T$ from (3.7) and (3.11) into (A.1) gives:

$$
\begin{aligned}
T C & =\frac{K+h_{i}\left(\frac{Q^{2}}{P} \sum_{n=0}^{\infty} \frac{\alpha^{n} Q^{n \beta}}{(2+n \beta)}+\frac{Q^{2-\beta}}{D(2-\beta)}\right)}{\sum_{n=0}^{\infty} \frac{\alpha^{n} Q^{n \beta+1}}{P(1+n \beta)}+\frac{Q^{1-\beta}}{D(1-\beta)}} \\
= & \frac{K+\sum_{n=0}^{\infty} \frac{h_{i} \alpha^{n} Q^{n \beta+2}}{P(2+n \beta)}+\frac{h_{i} Q^{2-\beta}}{D(2-\beta)}}{\sum_{n=0}^{\infty} \frac{\alpha^{n} Q^{n \beta+1}}{P(1+n \beta)}+\frac{Q^{1-\beta}}{D(1-\beta)}} .
\end{aligned}
$$

Setting the derivative of $T C$ with respect $Q=0$ :

$$
\begin{aligned}
T C^{\prime}= & \frac{\left(\sum_{n=0}^{\infty} \frac{h_{i} \alpha^{n} Q^{n \beta+1}}{P}+\frac{h_{i} Q^{1-\beta}}{D}\right)\left(\sum_{n=0}^{\infty} \frac{\alpha^{n} Q^{n \beta+1}}{P(1+n \beta)}+\frac{Q^{1-\beta}}{D(1-\beta)}\right)}{\left(\sum_{n=0}^{\infty} \frac{\alpha^{n} Q^{n \beta+1}}{P(1+n \beta)}+\frac{Q^{1-\beta}}{D(1-\beta)}\right)^{2}} \\
& -\frac{\left(K+\sum_{n=0}^{\infty} \frac{h_{i} \alpha^{n} Q^{n \beta+2}}{P(2+n \beta)}+\frac{h_{i} Q^{2-\beta}}{D(2-\beta)}\right)\left(\sum_{n=0}^{\infty} \frac{\alpha^{n} Q^{n \beta}}{P}+\frac{Q^{-\beta}}{D}\right)}{\left(\sum_{n=0}^{\infty} \frac{\alpha^{n} Q^{n \beta+1}}{P(1+n \beta)}+\frac{Q^{1-\beta}}{D(1-\beta)}\right)^{2}} \\
= & 0 .
\end{aligned}
$$

Since the numerator $=0$, then:

$$
\begin{aligned}
& \left(\sum_{n=0}^{\infty} \frac{h_{i} \alpha^{n} Q^{n \beta+1}}{P}+\frac{h_{i} Q^{1-\beta}}{D}\right)\left(\sum_{n=0}^{\infty} \frac{\alpha^{n} Q^{n \beta+1}}{P(1+n \beta)}+\frac{Q^{1-\beta}}{D(1-\beta)}\right) \\
& =\left(K+\sum_{n=1}^{\infty} \frac{h_{i} \alpha^{n} Q^{n \beta+2}}{P(2+n \beta)}+\frac{h_{i} Q^{2-\beta}}{D(2-\beta)}\right)\left(\sum_{n=0}^{\infty} \frac{\alpha^{n} Q^{n \beta}}{P}+\frac{Q^{-\beta}}{D}\right) .
\end{aligned}
$$


Dividing both sides by $\left(\sum_{n=0}^{\infty} \frac{\alpha^{n} Q^{n \beta}}{P}+\frac{Q^{-\beta}}{D}\right)$ and simplifying gives:

$$
\sum_{n=0}^{\infty} \frac{h \alpha^{n} Q^{n \beta+2}}{P(1+n \beta)(2+n \beta)}+\frac{h Q^{2-\beta}}{D(1-\beta)(2-\beta)}=K .
$$

Multiplying both sides by $\frac{D(1-\beta)(2-\beta)}{h}$ gives:

$$
(1-\beta)(2-\beta) \sum_{n=0}^{\infty} \frac{D \alpha^{n} Q^{n \beta+2}}{P(1+n \beta)(2+n \beta)}+Q^{2-\beta}=\frac{K D(1-\beta)(2-\beta)}{h} .
$$

Finally, substituting $\alpha=D / P$ in the above equation and rearranging leads to equation (3.12).

Acknowledgements. The author is grateful to the Fulbright Foundation for the funding of this research, and to the University of North Carolina, Charlotte, for the kind hospitality.

\section{REFERENCES}

[1] H.K. Alfares, Inventory model with stock-level dependent demand rate and variable holding cost. Int. J. Prod. Econ. 108 (2007) 259-265.

[2] R.C. Baker and T.L. Urban, A deterministic inventory system with an inventory level dependent rate. J. Oper. Res. Soc. 39 (1988a) 823-831.

[3] R.C. Baker and T.L. Urban, Single-period inventory dependent demand models. Omega-The Int. J. Manag. Sci. 16 (1988b) 605-607.

[4] M. Ferguson, V. Jayaraman and G.V. Souza, Note: An application of the EOQ model with nonlinear holding cost to inventory management of perishables. Eur. J. Oper. Res. 180 (2007) 485-490.

[5] M. Gayen and A.K. Pal, A two ware house inventory model for deteriorating items with stock dependent demand rate and holding cost. Oper. Res. 9 (2009) 153-165.

[6] B.C. Giri and K.S. Chaudhuri, Deterministic models of perishable inventory with stockdependent demand rate and nonlinear holding cost. Eur. J. Oper. Res. 105 (1998) 467-474.

[7] B.C Giri, A. Goswami and K.S. Chaudhuri, An EOQ model for deteriorating items with time-varying demand and costs. J. Oper. Res. Soc. 47 (1996) 1398-1405.

[8] M. Goh, Some results for inventory models having inventory level dependent demand rate. Int. J. Prod. Econ. 27 (1992) 155-160.

[9] M. Goh, EOQ models with general demand and holding cost functions. Eur. J. Oper. Res. 73 (1994) 50-54.

[10] R. Gupta and P. Vrat, Inventory model for stock-dependent consumption rate. Opsearch $\mathbf{2 3}$ (1986) 19-24.

[11] M.A. Koschat, Store inventory can affect demand: empirical evidence from magazine retailing. J. Retailing 84 (2008) 165-179.

[12] J. Min and Y.-W. Zhou, A perishable inventory model under stock-dependent selling rate and shortage-dependent partial backlogging with capacity constraint. Int. J. Syst. Sci. 40 (2009) 33-44.

[13] G.C. Mahata and A. Goswami, Fuzzy EOQ models for deteriorating items with stock dependent demand \& non-linear holding costs. Int. J. Appl. Math. Comput. Sci. 5 (2009) 94-98.

[14] A.P. Muhlemann and N.P. Valtis-Spanopoulos, Variable holding cost rate model. Eur. J. Oper. Res. 4 (1980) 132-135. 
[15] A. Roy, An inventory model for deteriorating items with price dependent demand and timevarying holding cost. Adv. Model. Optim. 10 (2008) 25-37.

[16] S.S. Sana, B.K. Sarkar, K. Chaudhuri and D. Purohit, The effect of stock, price and advertising on demand - An EOQ model. IJMIC 6 (2009) 81-88.

[17] A.R. Sarfaraz, A Deterministic and stochastic model for production lot size as a function of storage cost. Int. J. Modern Engrg. 9 (2009) 67-72.

[18] S.R. Singh, S. Kumar and N.K. Gaur, Inventory model with power demand, partial back logging, and incremental holding cost under inflation. Int. Trans. Appl. Sci. 1 (2009) 93-102.

[19] N. Singh, S.R. Singh and B. Vaish, EPQ model for time varying production and demand rate with trade credit. Asia-Pacific Operational Research Societies Conference (APORS 2009), Jaipur, India pp. 6-9.

[20] J.-T. Teng and H.-L. Yang, Deterministic inventory lot-size models with time-varying demand and cost under generalized holding costs. Inform. Manag. Sci. 18 (2007) 113-125.

[21] C.K. Tripathy, L.M. Pradhan and U. Mishra, An EPQ model for linear deteriorating item with variable holding cost. Int. J. Comput. Appl. Math. 5 (2010) 209-215.

[22] T.L. Urban, Inventory models with inventory-level-dependent demand: a comprehensive review and unifying theory. Eur. J. Oper. Res. 162 (2005) 792-804.

[23] T.L. Urban, An extension of inventory models with discretely variable holding costs. Int. J. Prod. Econ. 114 (2008) 399-403.

[24] H.J. Weiss, Economic order quantity models with nonlinear holding costs. Eur. J. Oper. Res. 9 (1982) 56-60.

[25] H.-L. Yang, J.-T. Teng and M.-S. Chern, An inventory model under inflation for deteriorating items with stock-dependent consumption rate and partial backlogging shortages. Int. J. Prod. Econ. 123 (2010) 8-19. 